\title{
Molecular Scale Buckling Mechanics in Individual Aligned Single-Wall Carbon Nanotubes on Elastomeric Substrates
}

\author{
Dahl-Young Khang,,$\S$ Jianliang Xiao,‡ Coskun Kocabas,, , $\S$ Scott MacLaren,,$\S$ \\ Tony Banks, ${ }^{\S}$ Hanqing Jiang," Yonggang Y. Huang, ${ }^{*}, \mathbb{I}$ and John A. Rogers ${ }^{*, t, \neq, \S}$
}

\begin{abstract}
Department of Materials Science and Engineering and Beckman Institute, University of Illinois at Urbana-Champaign, Urbana, Illinois 61801, Department of Mechanical Science and Engineering, University of Illinois at Urbana-Champaign, Urbana, Illinois 61801, Department of Physics, Beckman Institute, Frederick-Seitz Materials Research Laboratory, University of Illinois at Urbana-Champaign, Urbana, Illinois 61801, Frederick-Seitz Materials Research Laboratory, University of Illinois at Urbana-Champaign, Urbana, Illinois 61801, Department of Mechanical and Aerospace Engineering, Arizona State University, Tempe, Arizona 85287, and Departments of Civil and Environmental Engineering and Mechanical Engineering, Northwestern University, Evanston, Illinois 60208
\end{abstract}

Received August 30, 2007; Revised Manuscript Received November 5, 2007

\begin{abstract}
We have studied the scaling of controlled nonlinear buckling processes in materials with dimensions in the molecular range (i.e., $\sim 1 \mathrm{~nm}$ ) through experimental and theoretical studies of buckling in individual single-wall carbon nanotubes on substrates of poly(dimethylsiloxane). The results show not only the ability to create and manipulate patterns of buckling at these molecular scales, but also, that analytical continuum mechanics theory can explain, quantitatively, all measurable aspects of this system. Inverse calculation applied to measurements of diameterdependent buckling wavelengths yields accurate values of the Young's moduli of individual SWNTs. As an example of the value of this system beyond its use in this type of molecular scale metrology, we implement parallel arrays of buckled SWNTs as a class of mechanically stretchable conductor.
\end{abstract}

Controlled compression of structures that consist of high modulus layers on compliant supports can create, through nonlinear buckling processes, well-defined sinusoidal distributions of surface relief. ${ }^{1-14}$ This phenomena has attracted significant attention recently due to the utility of this type of mechanics in applications ranging from precision metrology $^{4,5}$ to stretchable electronics. ${ }^{9-14}$ Recent research demonstrates the ability to form diverse classes of buckled, wrinkled, bridging, and other structural configurations of nanomaterials (i.e., nanoscale membranes, ribbons, platelets, and related) by integrating them with compliant substrates

* To whom correspondence should be addressed. E-mail: y-huang@ northwestern.edu (Y.Y.H.); jrogers@uiuic.edu (J.A.R.).

$\dagger$ Department of Materials Science and Engineering, and Beckman Institute, University of Illinois at Urbana-Champaign.

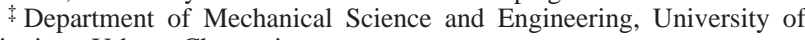
Illinois at Urbana-Champaign.

${ }^{£}$ Department of Physics, Beckman Institute, Frederick-Seitz Materials Research Laboratory, University of Illinois at Urbana-Champaign.

$\S$ Frederick-Seitz Materials Research Laboratory, University of Illinois at Urbana-Champaign.

\# Arizona State University

II Northwestern University. and applying mechanical forces, either before or after the nanomaterials and substrates are joined..$^{2-14}$ The engineering flexibility with which these configurations can be achieved, in parallel and over large areas, creates interest in the underlying physics and also in aspects of enabled applications in biotechnology, electronics, metrology, and other areas. We demonstrate here that this strategy can be used to manipulate materials with molecular scale (i.e., $\sim 1 \mathrm{~nm}$ ) dimensions and that Newtonian mechanics models can accurately describe the physics at these scales. The results include experimental and theoretical studies of the buckling, into sinusoidal "wavy" configurations, of individual linear SWNTs on elastomeric supports. The use of such data to determine Young's moduli for SWNTs and the implementation of wavy SWNTs as stretchable conductors provide application examples in molecular scale mechanics metrology and emerging electronic systems, respectively.

Figure 1a schematically describes the process for forming mechanically buckled, that is, "wavy", individual aligned SWNTs on an elastomeric substrate of poly(dimethylsilox- 
a

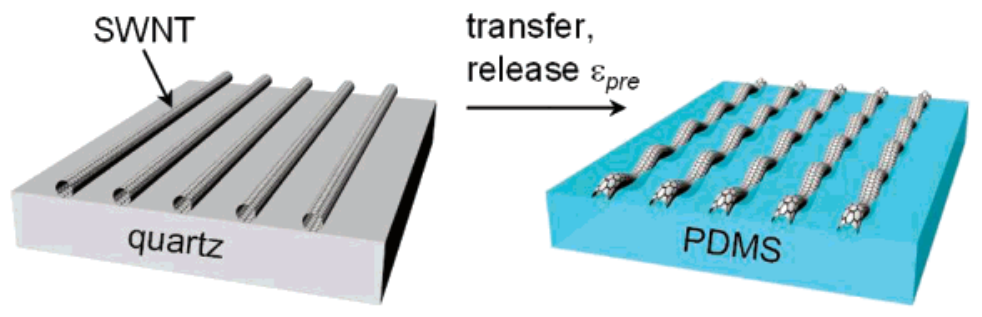

b
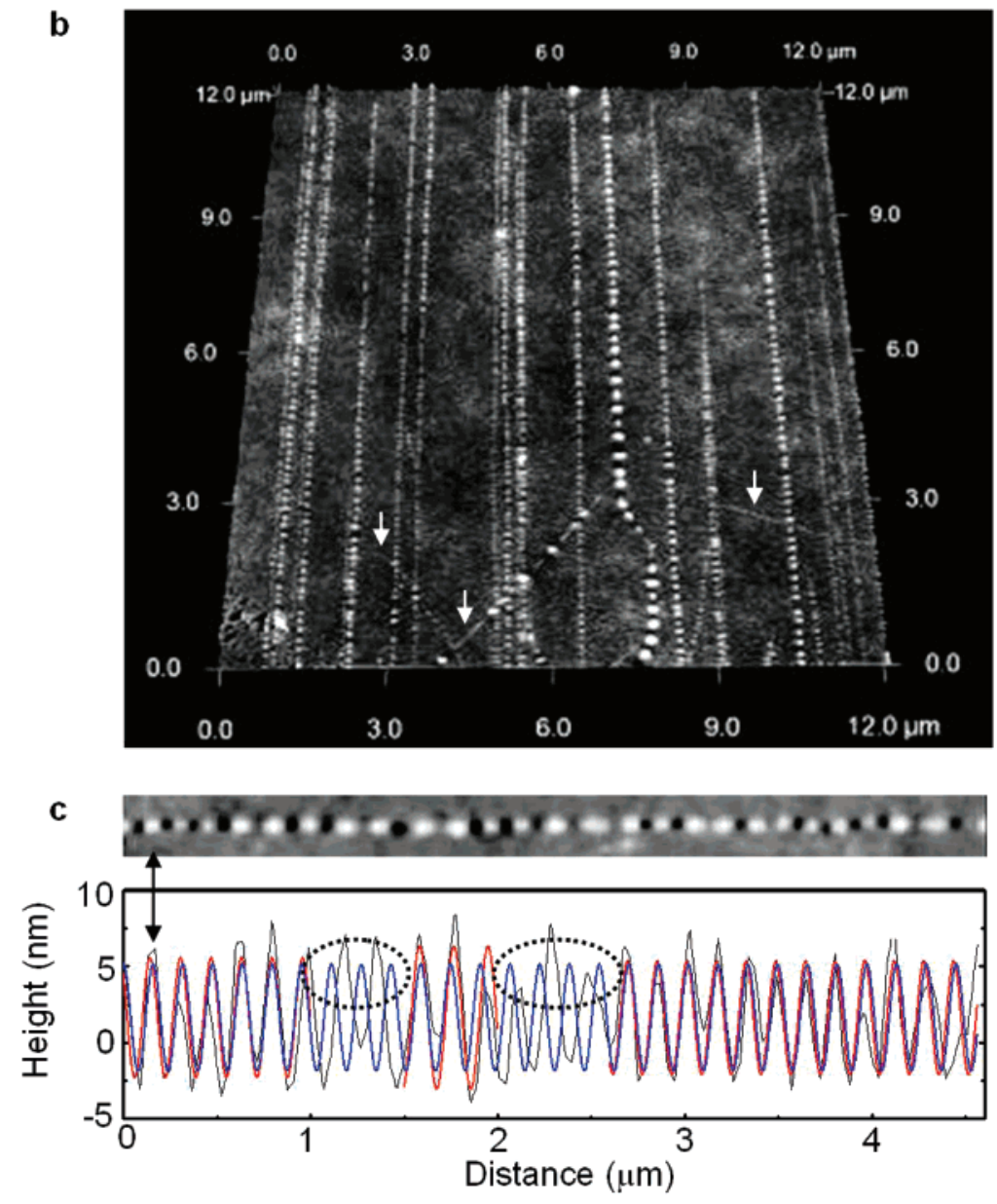

Figure 1. (a) Transfer of aligned arrays of SWNTs grown on quartz to a uniaxially strained substrate of PDMS followed by release of the prestrain $\left(\epsilon_{\text {pre }}\right)$ causes nonlinear buckling instabilities in the SWNT that lead to wavy configurations. (b) Large-area $(12 \mu \mathrm{m} \times 12 \mu \mathrm{m})$ angled-view atomic force microscope (AFM) image of wavy SWNTs on a PDMS substrate. (c) Plane-view AFM image of an individual wavy SWNT and linecut showing the profile of relief. The red, black, and blue curves represent measured data, a piecewise sinusoidal fit, and a global sinusoidal fit, respectively. The dashed ovals indicate regions that represent abrupt shifts in the phase. The wavelength determined by the piecewise fit is $160 \pm 20 \mathrm{~nm}$.

ane) (PDMS). Chemical vapor deposition growth on quartz substrates using optimized procedures ${ }^{15-17}$ formed arrays of aligned SWNTs with linear shapes and diameters between $\sim 1$ and $\sim 4 \mathrm{~nm}$. Aligned SWNTs on quartz substrates can be transferred to a mechanically prestrained (i.e., 3-5\% uniaxial tensile, $\epsilon_{\text {pre }}$, along the lengths of the SWNTs) PDMS substrate using modified versions of methods described in the literature. ${ }^{17,18}$ Releasing the prestrain creates, through a nonlinear buckling process, wavy SWNTs in which the heights of the SWNTs (and the underlying PDMS) vary periodically with position along their lengths. This configuration is much different from, but related to, recent reports of deformations in multiwalled carbon nanotube foams ${ }^{19}$ and random networks of SWNTs. ${ }^{20}$ Figure $1 \mathrm{~b}$ shows an angled view of wavy SWNTs collected with an atomic force microscope (AFM). The periods of the waves are between 100 and $300 \mathrm{~nm}$, with amplitudes of several nanometers. Three tubes (arrows) are misaligned with the prestrain and therefore do not form wavy structures. Figure 1c provides an AFM image and linecut along a representative wavy SWNT. The black, red, and blue curves correspond to the AFM data, a piecewise sinusoidal fit, and a global sinusoidal fit, respectively. The data exhibit a single spatial frequency, separated by phase jumps at certain locations (dotted ovals). The sinusoidal shapes are qualitatively consistent with expectations based on much larger scale membrane and ribbon structures. ${ }^{2-14}$ The wavelength for this case is $\sim 160$ $\mathrm{nm}$, evaluated from the fitting. The phase shifts represent 
a

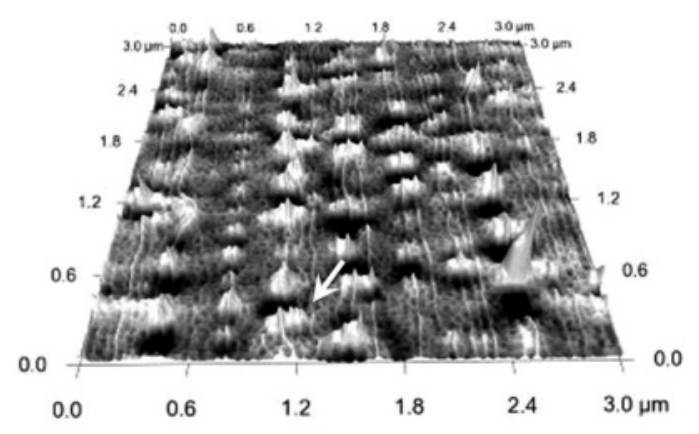

b

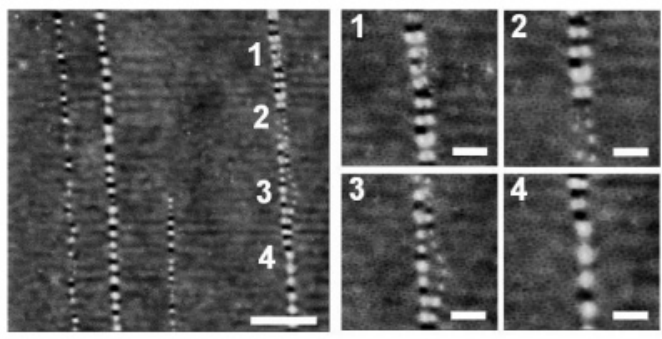

C

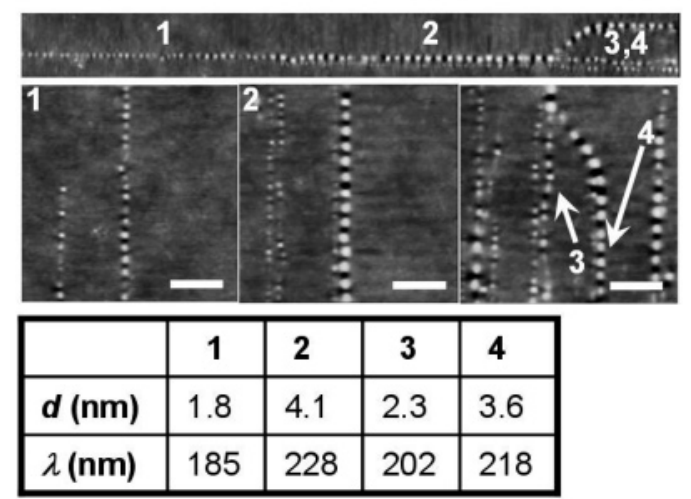

Figure 2. (a) High-resolution atomic force microscope (AFM) images of wavy SWNTs, highlighting mechanical coupling, variations in amplitude and wavelength, and absence of buckling in certain SWNTs. (b) AFM images that illustrate mechanical coupling. When two or more SWNTs are sufficiently close, they adopt the same wavelength and phase $(\mathbf{1}, \mathbf{4})$. For larger separations, each SWNTs exhibits its own buckling properties $(2, \mathbf{3})$. The critical separation distance for coupling is observed to be $\sim 70-100 \mathrm{~nm}$. Scale bars are $1 \mu \mathrm{m}$ (left) and $300 \mathrm{~nm}$ (right); the difference in height between the darkest and lightest regions is $20 \mathrm{~nm}$ for all images. (c) AFM images that illustrate the dependence of the wavelength and amplitude on SWNT diameter. Bundles or close clusters of SWNTs lead to spatial variations in the wavelength and amplitude with length. The scale bars are $1 \mu \mathrm{m}$; the difference in height between the darkest and lightest regions is $20 \mathrm{~nm}$ for all images.

nonideal features that are likely due either to local variations in the properties of the PDMS or to regions where the SWNT slipped along the surface of the PDMS during the buckling process.

These and other aspects of the system can be seen more clearly in the images of Figure 2. Figure 2a shows a highresolution AFM image collected in a region with a large number $(\sim 30)$ of SWNTs. Three features are notable. First, not all of the SWNTs adopt a wavy shape. The SWNT highlighted with the arrow, for example, lies flat on the PDMS and is not straight. Since the growth process produces almost perfect yields of linear SWNTs, we suspect that this tube slipped on the surface of the PDMS during the release of the prestrain or during transfer, thereby avoiding the buckling process and yielding a nonlinear configuration. A second feature is that SWNTs with wavy shapes have welldefined amplitudes and wavelengths; these parameters can vary from one SWNT to the next across the substrate. Finally, when they are sufficiently close to one another, the wavy SWNTs adopt common wavelengths and phases. This type of coupling, which is mechanically mediated by the PDMS, can be clearly seen in many parts of Figure $2 a$. Figures $2 b$ shows additional examples of coupling. Figure $2 c$ illustrates the diameter dependence of the wavelength in a single SWNT structure that, we speculate, consists of an individual tube (1; left) that transitions to a small bundle of SWNTs (likely 2) $(2$; middle) and then to a branching set of SWNTs (3, 4; right).

All of these behaviors can be captured quantitatively with a Newtonian analytical mechanics model that exploits linear elasticity theory. ${ }^{21,22} \mathrm{We}$ approximate the SWNT as a hollow, elastic tube with outer radius $R$, thickness $t$, and Young's modulus $E_{\mathrm{CNT}}$. The PDMS substrate is modeled as a semiinfinite solid with Young's modulus $E_{\mathrm{S}}$ and Poisson's ratio $v_{\mathrm{S}}$. The out-of-plane displacement of a wavy SWNT takes a sinusoidal form along the tube direction, with a height that varies according to $w=A \cos (k x)$, where $A$ is the amplitude and $k$ is the wavevector, with $\lambda=2 \pi / k$. The total energy of the system consists of three parts, the strain energy in the PDMS substrate and the membrane and bending energies of the SWNT. Each part can be obtained analytically in terms of $A$ and $k$. Minimizing the total system energy with respect to $A$ and $k$ yields the following expressions for the wavevector and amplitude

$$
\begin{gathered}
k\left(\frac{E_{\mathrm{CNT}} I}{\bar{E}_{\mathrm{S}}}\right)^{1 / 4}=\left[\frac{2 \pi(1-\gamma-\ln k R)}{(3-2 \gamma-2 \ln k R)^{2}}\right]^{1 / 4} \\
A=\frac{2}{k}\left[\epsilon_{\mathrm{pre}}-\epsilon_{\mathrm{a}}-\frac{E_{\mathrm{CNT}} I}{E_{\mathrm{CNT}} S} k^{2}-\frac{\bar{E}_{\mathrm{S}} \pi}{E_{\mathrm{CNT}} S k^{2}(3-2 \gamma-2 \ln k R)}\right]^{1 / 2}
\end{gathered}
$$

Here, $\gamma=0.577$ is Euler's constant, $\bar{E}_{\mathrm{S}}=\left(E_{\mathrm{S}} /\left(1-v_{\mathrm{S}}^{2}\right)\right)$ is the plane-strain modulus of the PDMS substrate, and $E_{\mathrm{CNT}} I$ and $E_{\mathrm{CNT}} S$ are the bending stiffness and tension stiffness of the carbon nanotube, respectively. The quantities $\epsilon_{\text {pre }}$ and $\epsilon_{\mathrm{a}}$ correspond to the prestrain and to strain applied to the system after its fabrication, respectively. In the small-deformation limit used to derive eqs 1 and 2, the value of $k$ (and, therefore, $\lambda$ ) is independent of the applied strain. Equation 1 for the wavenumber has the following simple approximate (to within $\sim 5 \%)$ solution when $R>0.5 \mathrm{~nm}$ and $E_{\mathrm{CNT}} / \bar{E}_{\mathrm{S}}>25000$

$$
k=\frac{3}{4}\left(\frac{\bar{E}_{\mathrm{S}}}{E_{\mathrm{CNT} I}}\right)^{1 / 4}
$$

In this case, $A$ can be written 


$$
\begin{aligned}
A=2\left[\frac{16}{9}\left(\frac{E_{\mathrm{CNT}} I}{\bar{E}_{\mathrm{S}}}\right)^{1 / 2}\left(\epsilon_{\mathrm{pre}}-\epsilon_{\mathrm{a}}\right)-\right. \\
\\
\left.\quad \frac{E_{\mathrm{CNT} I} I}{E_{\mathrm{CNT}} S}\left(1+\frac{256 \pi}{81(3-2 \gamma-2 \ln k R)}\right)\right]^{1 / 2}
\end{aligned}
$$

The critical prestrain, $\epsilon_{\text {critical }}$, which corresponds to the smallest $\epsilon_{\text {pre }}$ that leads to buckling can be obtained from $A$ $=0$ as

$$
\epsilon_{\text {critical }}=\frac{9}{16} \frac{\sqrt{E_{\mathrm{S}} E_{\mathrm{CNT}} I}}{E_{\mathrm{CNT}} S}\left(1+\frac{256 \pi}{81(3-2 \gamma-2 \ln k R)}\right)
$$

These expressions for $A$ and $k$ are valid not only for individual SWNTs but also for multiwalled nanotubes and bundles of SWNTs, provided that $E_{\mathrm{CNT}} I, E_{\mathrm{CNT}} S$, and $R$ are replaced by the corresponding bending stiffness, tension stiffness, and outer radius, respectively.

For an individual SWNT, the moment of inertia, $I$, is $\pi R^{3} t$, and the area $S$ is $2 \pi R t$, where $R$ is the outer radius of the SWNT and $t$ is its wall thickness. The bending stiffness $E_{\mathrm{CNT}} I$ and tension stiffness $E_{\mathrm{CNT}} S$ in eqs $1-5$ then become $E_{\mathrm{CNT}} t^{\circ}$ $\pi R^{3}$ and $E_{\mathrm{CNT}} t \cdot 2 \pi R$, respectively. It is interesting to observe that the SWNT Young's modulus $E_{\mathrm{CNT}}$ and thickness $t$ always appear together via their product $E_{\mathrm{CNT}} t$. Huang et al. ${ }^{23}$ showed that it is unnecessary to define the SWNT Young's modulus and thickness separately since all experimentally measurable or theoretically calculable properties involve $E_{\mathrm{CNT}} t$, not $E_{\mathrm{CNT}}$ nor $t$ separately. In fact, the prior modeling and simulations of SWNTs can be grouped into two types; one takes the interlayer spacing of graphite, $0.34 \mathrm{~nm}$, as the SWNT thickness, and the resulting Young's modulus is around $1 \mathrm{TPa} ;{ }^{24-27}$ and the other is based on the continuum shell modeling, which gives the thickness around $0.066 \mathrm{~nm}$ and Young's modulus 5.5 TPa. ${ }^{28-33}$ Their two types, however, give approximately the same $E_{\mathrm{CNT}} t, 0.34 \mathrm{TPa} \cdot \mathrm{nm}$ for the first group and $0.36 \mathrm{TPa} \cdot \mathrm{nm}$ for the second group. In this letter, we take the interlayer spacing of graphite, 0.335 $\mathrm{nm}$, as the SWNT thickness. ${ }^{34}$ For this case, the value of $\epsilon_{\text {critical }}$, using $\bar{E}_{\mathrm{S}}=2.6 \mathrm{MPa}, E_{\mathrm{CNT}}=1.3 \mathrm{TPa}$, and $R=1 \mathrm{~nm}$, is $0.08 \%$, which is much lower than $\epsilon_{\text {pre }}$ used in the experiments presented here. The computed amplitudes are around $5 \mathrm{~nm}$, consistent with those observed experimentally (Figure 1). Figure 3a shows angled views of computed displacements in a system with a layout chosen to correspond approximately to experiment. The displacements in the PDMS decay away from the SWNT, and for distances larger than $\sim 50 \mathrm{~nm}$, the effect of the SWNT disappears. The SWNTs buckle independently until their spacing decreases to a critical value, determined by equating the sum of the energy for two individual SWNTs (and the substrate) to the energy for two SWNTs that interact via the substrate (see the Supporting Information). For SWNT spacings less than the critical value, the SWNTs buckle together and adopt the same wavelength and phase. This critical spacing depends on the dimensions of the SWNTs but is typically in the range of $50 \mathrm{~nm}$ for the SWNTs investigated here. For example, for two SWNTs with radii $R_{1}=1 \mathrm{~nm}$ and $R_{2}=1.1 \mathrm{~nm}$, the

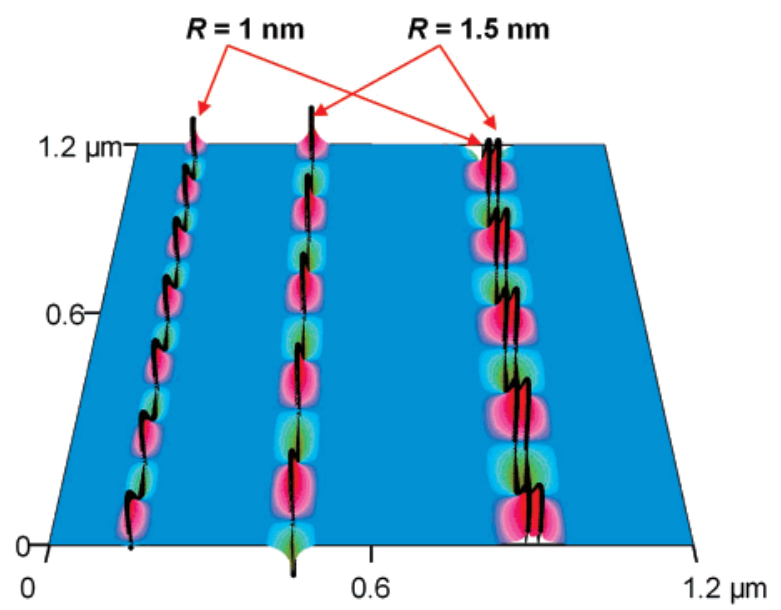

b

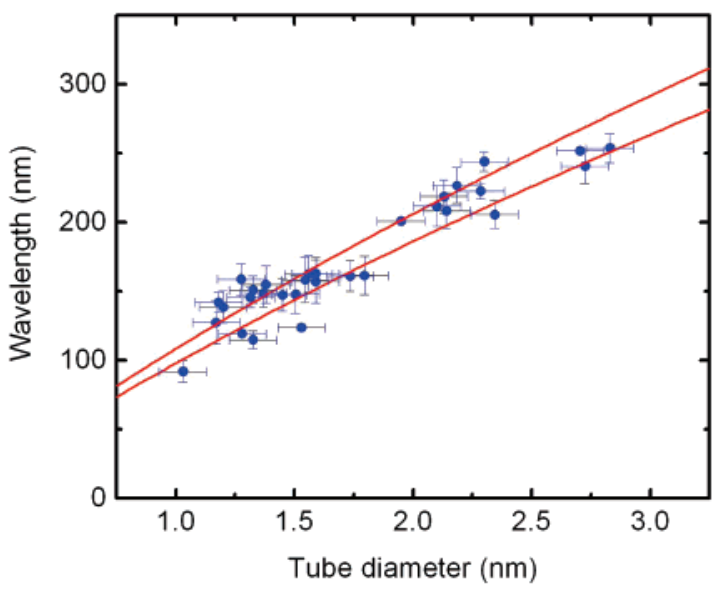

Figure 3. (a) Calculated deformations in isolated (left) and mechanically coupled (right) SWNTs on PDMS, rendered in a threedimensional perspective image. The effects of diameter-dependent wavelengths and mechanical coupling can be seen clearly. (b) Comparison between experimentally measured buckling wavelengths and calculation (lines) in which the Young's modulus of the SWNTs, $E_{\mathrm{CNT}}$, serves as a single variable parameter. The upper and lower curves correspond to a modulus of 1.5 and $1.1 \mathrm{TPa}$, respectively. On the basis of these results, we conclude that the modulus is $1.3 \pm 0.2 \mathrm{TPa}$.

spacing is $52 \mathrm{~nm}$. For $R_{1}=1 \mathrm{~nm}$ and $R_{2}=1.5 \mathrm{~nm}$, the spacing decreases to $37 \mathrm{~nm}$. Critical spacings estimated from experiment are $\sim 70-100 \mathrm{~nm}$, depending on tube diameter. When the SWNTs couple mechanically, they adopt a wavelength very close to that of the larger tube when it buckles in isolation. These predictions are consistent with experiment.

Another valuable insight obtained from computations is that normal forces at the interface between a SWNT and the PDMS, for parameters comparable to those in experiment, are smaller than typical van der Waals attractive forces. In particular, the normal force (per unit length) at the SWNT/ PDMS interface is on the order of $0.01 \mathrm{nN} / \mathrm{nm}$ (see the Supporting Information), which is much less than the van der Waals force of $0.16 \mathrm{nN} / \mathrm{nm}$ for a SWNT with a $2 \mathrm{~nm}$ diameter. ${ }^{35}$ These calculations explain, then, the observation that most SWNT buckle, rather than delaminate or slip, 
a
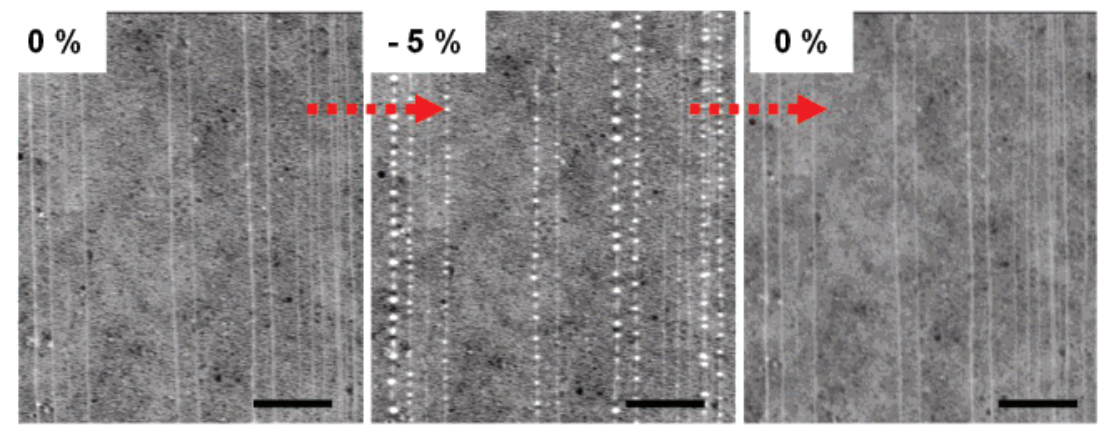

b
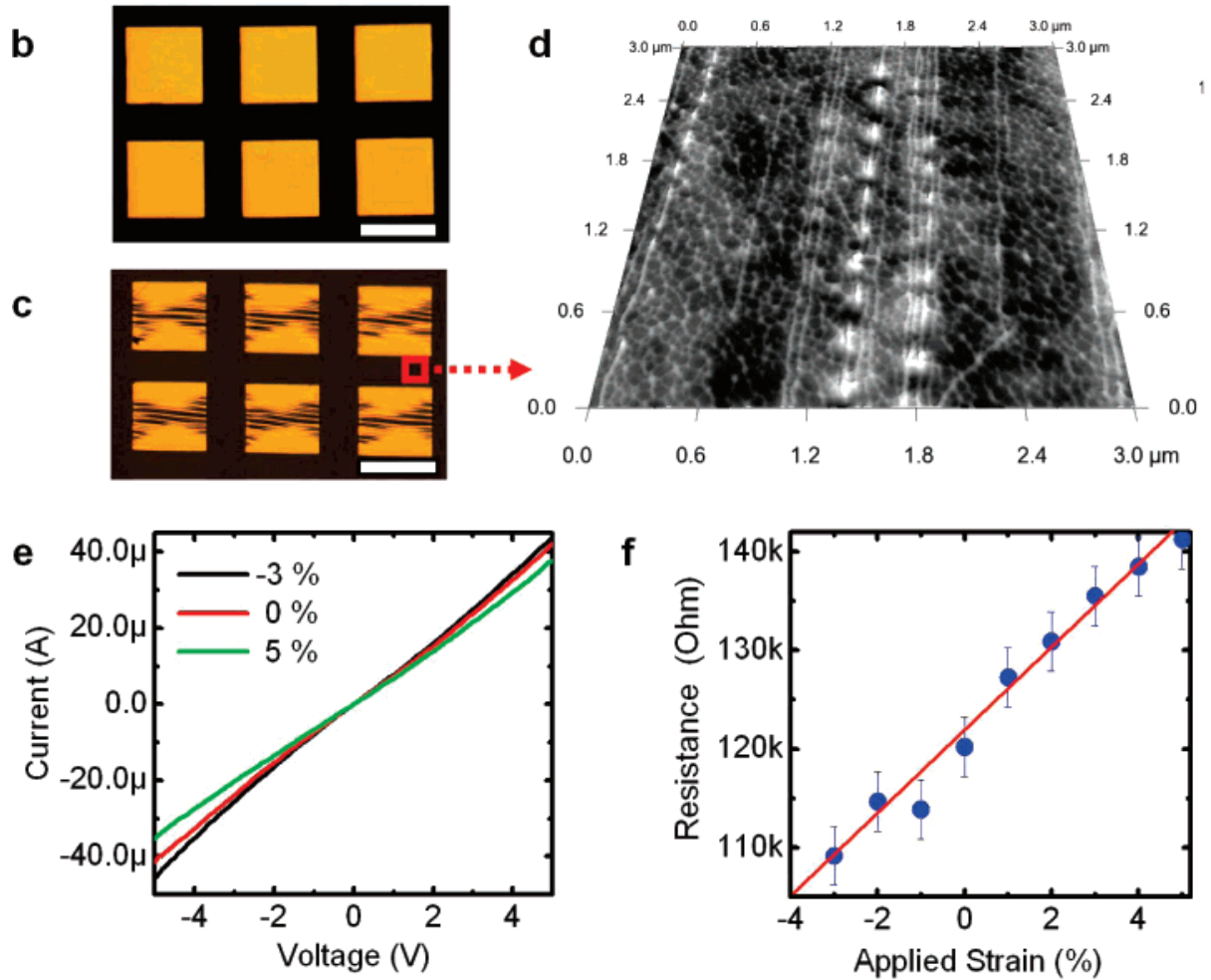

Figure 4. (a) Atomic force microscope (AFM) images in a single region of a sample prepared with $\epsilon_{\text {pre }}=0 \%$, collected before (left), during (center), and after (right) application of 5\% compressive strain. The system shows completely reversible behavior for this range of strains. Scale bars are $1 \mu \mathrm{m}$. (b,c) Optical microscope images of arrays of two terminal devices on PDMS, formed with $\epsilon_{\text {pre }}=0$ and $5 \%$, respectively. Scale bars are $80 \mu \mathrm{m}$. (d) AFM image of wavy SWNTs in the region between the pair of electrodes, indicated with a red box in c. (e) Current-voltage curves for a representative device, measured at various values of applied strain, for a sample prepared with $\epsilon_{\text {pre }}$ $=5 \%$. (f) Change of the resistance of a device as a function of applied strain. The gauge factor is $\sim 4$.

during the release of $\epsilon_{\text {pre }}$, despite the absence of any specific chemical interactions between the SWNTs and the PDMS.

Fitting the measured dependence of $\lambda$ on $d$ to the expression in eq 1 can yield the Young's modulus of the SWNTs. Figure $3 \mathrm{~b}$ shows the results, in which the upper and lower lines correspond to calculations where the modulus is 1.5 and $1.1 \mathrm{TPa}$, respectively, allowing us to conclude that $E_{\mathrm{CNT}}=1.3 \pm 0.2 \mathrm{TPa}$. This result is in quantitative agreement with the only previous report of a measurement of the Young's modulus in an individual isolated SWNT, as determined by atomic force measurements on a single suspended SWNT. ${ }^{36}$ The values are also consistent with firstprinciples calculations,${ }^{37-39}$ as well as moduli measured for bundles of SWNTs and for multiwalled nanotubes, as determined from vibrating reed and related types of experiments (none of which is suited to the measurement of isolated individual SWNTs). ${ }^{40-48}$ The calculated Young's modulus of a SWNT is linearly sensitive to the modulus of the PDMS because the results of the calculations depend on the ratio $E_{\mathrm{CNT}} / \bar{E}_{\mathrm{S}}$, that is, the value will increase or decrease by $10 \%$ if the modulus of PDMS increases or decreases by $10 \%$. We used the wavelength information for evaluating the modulus because the wavelength depends less strongly on the value of the prestrain than the amplitude. Also, the wavelength, unlike the amplitude, is relatively insensitive to the residual surface roughness on the PDMS and can therefore be measured with greater accuracy. The models and the fitting can also lead to conclusions on the nature of the tubes. For example, if we assume the nanotubes are double-walled, triple-walled, or small bundles, then appropriate models (i.e., values for $I$ and $S$ ) lead to fitted values for the Young's moduli of $\sim 1.0 \mathrm{TPa}$ (using data with diameters $>1.36 \mathrm{~nm}$ ), $\sim 0.9 \mathrm{TPa}$ (using data with diameters $>2.04 \mathrm{~nm}$ ), and $\sim 4.0$ $\mathrm{TPa}$ (using data with diameters $>1.28 \mathrm{~nm}$ ), respectively. These results suggest that most of the data of Figure $3 \mathrm{~b}$ likely correspond to individual SWNTs (see the Supporting Infor- 
mation). Collectively, the success of the models developed here in describing the experimental observations indicates that Newtonian mechanics provides accurate descriptions of these systems, even when macroscopic concepts such as tube wall thicknesses are atomic in dimension and where the mechanical properties of the PDMS, for which the average distance between cross-links is $1-2 \mathrm{~nm}$, are not well defined at nanometer length scales. The relatively long dimensions of the tubes, their high levels of structural perfection, and the small deformations involved in these experiments might help to explain the success of this model. Newtonian mechanics is known to be valid down to length scales corresponding to roughly 10 atomic spacings, for defectfree materials. ${ }^{49,50}$ It is important to note that SWNTs with diameters larger than $\sim 4 \mathrm{~nm}$ are susceptible to self-collapse. ${ }^{51}$ For this reason, and because SWNTs with apparent diameters larger than $\sim 3 \mathrm{~nm}$ are likely bundles of SWNTs (e.g., Figure 2c) or multiwalled tubes, we did not extend the analysis of the modulus to diameters larger than $3 \mathrm{~nm}$.

The inclusion of $\epsilon_{\mathrm{a}}$ into the analysis of eqs $1-5$ enables the straightforward application of the results to a system subjected to mechanical strains applied after release of $\epsilon_{\text {pre }}$. In experiments of this type, we observed fully reversible behavior, in which applied tensile (compressive) strains decreased (increased) A. Although we intuitively expected increases (reductions) in $\lambda$, changes in this quantity were too small to measure accurately over the range of applied strains explored here. (The theoretical prediction of eqs 1 and 3 that $\lambda$ does not vary with $\epsilon_{\mathrm{a}}$ or $\epsilon_{\text {pre }}$ arises from approximations associated with the small deformation limit. Full treatments, for thin films on compliant substrates, recover the expected dependencies of $\lambda$ on $\epsilon_{\mathrm{a}}$ or $\epsilon_{\mathrm{pre}}$, with functional forms that agree qualitatively with the response of an accordion bellows..$^{52}$ ) These variations in wavy shapes were reversible for tensile strains less than $\epsilon_{\text {pre }}$ and compressive strains less than $\sim 5 \%$. For these applied strains, theory suggests that mechanical forces do not exceed van der Waals attractions, and the SWNTs should not delaminate from the PDMS. Figure 4a shows AFM images collected at a single location on the substrate for which $\epsilon_{\text {pre }}=0 \%$. The numbers in the insets correspond to the values of $\epsilon_{\mathrm{a}}$. This reversible response with strain can be exploited to yield strain gauges, stretchable conductors/semiconductors, and related electronic components similar in concept to those that have been achieved with thin films of metals ${ }^{12,14}$ and single crystalline semiconductor ribbons. ${ }^{9-11,13}$ To demonstrate the basic effects, we built two terminal devices on SWNT/PDMS substrates by evaporating thin films of $\operatorname{Ti}(2 \mathrm{~nm}) / \mathrm{Au}(60 \mathrm{~nm})$ through shadow masks, in the prestrained state, to form electrode pairs with separations smaller than the average lengths of the SWNTs. Figure $4 \mathrm{~b}$ and $\mathrm{c}$ show optical micrographs of arrays of devices at $\epsilon_{\mathrm{a}}=0 \%$, formed in this manner, with $\epsilon_{\text {pre }}=0 \%$ (as that for Figure $4 \mathrm{a}$ ) and with $\epsilon_{\text {pre }}$ $=5 \%$ (SWNTs in a wavy state at $\epsilon_{\mathrm{a}}=0 \%$ ), respectively. Note the wavy metal electrodes in the case of Figure 4c. Figure 4d presents an AFM image of the wavy SWNTs in the region between an electrode pair (Figure 4c; red box). The average tube density is $\sim 2-3 \mathrm{SWNT} / \mu \mathrm{m}$. Figure $4 \mathrm{e}$ illustrates current/voltage measurements on a representative device, recorded at several values of $\epsilon_{\mathrm{a}}$, indicating that the resistance decreases with compression. The resistance of the device as a function of $\epsilon_{\mathrm{a}}$ is given in Figure 4f. A piezoresistance gauge factor $(\mathrm{GF})$ can be defined as $\mathrm{GF}=$ $(\Delta R / R) / \Delta \epsilon_{\mathrm{a}}$, where $R$ and $\Delta R$ denote the resistance and change in resistance with a change in the applied strain $\Delta \epsilon_{\mathrm{a}}$, respectively. The GF values (between $\sim 3$ and $\sim 8$ for samples prepared in this fashion and evaluated in this range of strains) are much smaller than that for a single isolated SWNT subjected to applied strains; ${ }^{36}$ they are similar to those observed in random networks of SWNTs. ${ }^{20}$ Although the complexity of the system (e.g., the response can be effected by strain-induced changes in the properties of the contacts) makes quantitative analysis difficult, we speculate that the relatively small values of GF in buckled systems, evaluated on length scales that are long compared to $\lambda$, arise, at least in part, from the compensating effects of the spatially alternating regions of compression and tension associated with the wavy geometries.

In summary, the results presented here illustrate the possibility of mechanically manipulating material structures with molecular scale dimensions (thickness and width). Its use in molecular scale metrology, as demonstrated here with SWNTs, could be useful for investigations of other similar types of materials, such as DNA and RNA. Electronics applications include not only stretchable conductors but other more sophisticated devices that involve strain coupling to electronic properties. These possibilities, together with the development of methods for enhanced engineering control over the buckled geometries through patterns of relief or surface chemistry on the elastomer, appear to represent promising directions for future research.

Acknowledgment. We thank K. Colravy for help with processing using facilities at the Frederick Seitz Materials Research Laboratory. This material is based upon work supported by the National Science Foundation under grants NIRT-0403489 and DMI-0328162, the U.S. Department of Energy, Division of Materials Sciences under Award No. DEFG02-91ER45439, through the Frederick Seitz MRL and Center for Microanalysis of Materials at the University of Illinois at Urbana-Champaign. HJ acknowledges the financial support from the Fulton School of Engineering at ASU.

Supporting Information Available: Additional experimental and theoretical details. This material is available free of charge via the Internet at http://pubs.acs.org.

\section{References}

(1) Allen, H. G. Analysis and Design of Structural Sandwich Panels; Pergamon: New York, 1969.

(2) Bowden, N.; Brittain, S.; Evans, A. G.; Hutchinson, J. W.; Whitesides, G. M. Nature 1998, 393, 146-149.

(3) Huck, W. T. S.; Bowden, N.; Onck, P.; Pardoen, T.; Hutchinson, J. W.; Whitesides, G. M. Langmuir 2000, 16, 3497-3501.

(4) Stafford, C. M.; Harrison, C.; Beers, K. L.; Karim, A.; Amis, E. J.; Vanlandingham, M. R.; Kim, H. C.; Volksen, W.; Miller, R. D.; Simonyi, E. E. Nat. Mater. 2004, 3, 545-550.

(5) Nolte, A. J.; Rubner, M. F.; Cohen, R. E. Macromolecules 2005, 38, 5367-5370. 
(6) Huang, Z. Y.; Hong, W.; Suo, Z. J. Mech. Phys. Solids 2005, 53, $2101-2118$

(7) Chen, X.; Hutchinson, J. W. J. Appl. Mech. 2004, 71, 597-603.

(8) Efimenko, K.; Rackaitis, M.; Manias, E.; Vaziri, A.; Mahadevan, L.; Genzer, J. Nat. Mater. 2005, 4, 293-297.

(9) Sun, Y.; Rogers, J. A. J. Mater. Chem. 2007, 17, 832-840.

(10) Sun, Y.; Choi, W. M.; Jiang, H.; Huang, Y. Y.; Rogers, J. A. Nat. Nanotechnol. 2006, 1, 201-207.

(11) Sun, Y.; Kumar, V.; Adesida, I.; Rogers, J. A. Adv. Mater. 2006, 18, 2857-2862.

(12) Lumelsky, V. J.; Shur, M. S.; Wagner, S. IEEE Sens. J. 2001, 1, $41-51$.

(13) Khang, D. Y.; Jiang, H. Q.; Huang, Y.; Rogers, J. A. Science 2006, $311,208-212$.

(14) Lacour, S. P.; Jones, J.; Wagner, S.; Li, T.; Suo, Z. Proc. IEEE 2005, 93, 1459-1467.

(15) Kocabas, C.; Hur, S.-H.; Gaur, A.; Meitl, M.; Shim, M.; Rogers, J. A. Small 2005, 1, 1110-1116.

(16) Kocabas, C.; Shim, M. S.; Rogers, J. A. J. Am. Chem. Soc. 2006 , $128,4540-4541$

(17) Kang, S. J.; Kocabas, C.; Ozel, T.; Shim, M.; Pimparkar, N.; Alam, M. A.; Rotkin, S. V.; Rogers, J. A. Nat. Nanotechnol. 2007, 2, 230236

(18) Kang, S. J.; Kocabas, C.; Kim, H.-S.; Cao, Q.; Meitl, M. A.; Khang, D.-Y.; Rogers, J. A. Nano Lett. 2007, 7, 3343-3348.

(19) Cao, A. Y.; Dickrell, P. L.; Sawyer, W. G.; Ghasemi-Nejhad, M. N.; Ajayan, P. M. Science 2005, 310, 1307-1310.

(20) Maune, H.; Bockrath, M. Appl. Phys. Lett. 2006, 89, 173131/1$173131 / 3$.

(21) Timoshenko, S. P. Strength of Materials; Van Nostrand: New York, 1941; Chapters 3-5.

(22) Timoshenko, S. P.; Goodier, J. N. Theory of Elasticity; McGrawHill: New York, 1970; Chapter 12.

(23) Huang, Y.; Wu, J.; Hwang, K. C. Phys. Rev. B 2006, 74, 245413/ $1-245413 / 9$.

(24) Lu, J. P. Phys. Rev. Lett. 1997, 79, 12971-12974.

(25) Hernández, E.; Goze, C.; Bernier, P.; Rubio, A. Phys. Rev. Lett. 1998, $80,4502 / 1-4502 / 4$.

(26) Li, C.; Chou, T. Int. J. Solid Struct. 2003, 40, 2487-2499.

(27) Jin, Y.; Yuan, F. G. Compos. Sci. Technol. 2003, 63, 1507-1515.

(28) Yakobson, B. I.; Brabec, C. J.; Bernholc, J. Phys. Rev. Lett. 1996, 76, 2511/1-2511/4.

(29) Zhou, X.; Zhou, J. J.; Ou-Yang, Z. C. Phys. Rev. B 2000, 62, 1369213696.
(30) Kudin, K. N.; Scuseria, G. E.; Yakobson, B. I. Phys. Rev. B 2001, $64,235406 / 1-235406 / 10$.

(31) Tu, Z.; Ou-Yang, Z. Phys. Rev. B 2002, 65, 233407/1-233407/4.

(32) Pantano, A.; Boyce, M. C.; Parks, D. M. Phys. Rev. Lett. 2003, 91, 145504/1-145504/4

(33) Wang, L.; Zheng, Q.; Liu, J. Z.; Jiang, Q. Phys. Rev. Lett. 2005, 95, $10550 / 1-10550 / 4$.

(34) Girifalco, L. A.; Lad, R. A. J. Chem. Phys. 1956, 25, 693-697.

(35) Jiang, L. Y.; Huang, Y.; Jiang, H.; Ravichandran, G.; Gao, H.; Hwang, K. C.; Liu, B. J. Mech. Phys. Solids 2006, 54, 2436-2452.

(36) Tombler, T. W.; Zhou, C.; Alexseyev, L.; Kong, J.; Dai, H.; Liu, L.; Jayanthi, C. S.; Tang, M.; Wu, S. Y. Nature 2000, 405, 769-772.

(37) Lier, G. V.; Alsenoy, C. V.; Doran, V. V.; Geerlings, P. Chem. Phys Lett. 2000, 326, 181-185.

(38) Sánchez-Portal, D.; Artacho, E.; Soler, J. M.; Rubio, A.; Ordejón, P. Phys. Rev. B 1999, 59, 12678-12688.

(39) Zhou, G.; Duan, W.; Gu, B. Chem. Phys. Lett. 2001, 333, 344-349.

(40) Treacy, M. M. J.; Ebbesen, T. W.; Gibson, J. M. Nature 1996, 381, 678-680.

(41) Wong, E. W.; Sheehan, P. E.; Lieber, C. M. Science 1997, 277, 1971-1975.

(42) Lourie, O.; Wagner, H. D. J. Mater. Res. 1998, 13, 2418-2422.

(43) Pan, Z. W.; Xie, S. S.; Lu, L.; Chang, B. H.; Sun, L. F.; Zhou, W. Y.; Wang, G. Appl. Phys. Lett. 1999, 74, 3152-3154.

(44) Yu, M.-F.; Lourie, O.; Dyer, M. J.; Moloni, K.; Kelly, T. F.; Ruoff, R. S. Science 2000, 287, 637-640.

(45) Krishnan, A.; Dujardin, E.; Ebbesen, T. W.; Yianilos, P. N.; Treacy, M. M. J. Phys. Rev. B 1998, 58, 14013-14019.

(46) Muster, J.; Burghard, M.; Roth, S.; Duesberg, G. S.; HernMandez, E.; Rubio, A. J. Vac. Sci. Technol., B 1998, 16, 2796-2801.

(47) Salvetat, J.-P.; Briggs, G. A. D.; Bonard, J.-M.; Bacsa, R. R.; Kulik, A. J. Phys. Rev. Lett. 1999, 82, 944-947.

(48) Poncharal, P.; Wang, Z. L.; Ugarte, D.; de Heer, W. A. Science 1999, $283,1513-1516$.

(49) Zhang, P.; Huang, Y.; Geubelle, P. H.; Klein, P. A.; Hwang, K. C. Int. J. Solids Struct. 2002, 39, 3893-3906.

(50) Jiang, H.; Zhang, P.; Liu, B.; Huang, Y.; Geubelle, P. H.; Gao, H.; Hwang, K. C. Comput. Mater. Sci. 2003, 28, 429-442.

(51) Liu, B.; Yu, M. F.; Huang, Y. Phys. Rev. B 2004, 70, 161402/1161402/4(R).

(52) Jiang, H.; Khang, D.-Y.; Song, J.; Sun, J.; Huang, Y.; Rogers, J. A Proc. Nat. Acad. Sci. USA 2007, 104, 15607-15612.

NL072203S 